\title{
COMUNICAÇÃOCIENTÍFICA \\ ENRAIZAMENTO DE ESTACAS APICAIS LENHOSAS \\ DE FIGUEIRA 'Roxo de Valinhos' COM APLICAÇÃO DE AIB E CIANAMIDA HIDROGENADA ${ }^{1}$
}

\author{
TATIANE OHLAND ${ }^{2}$, RAFAEL PIO $^{3}$, EDVAN ALVES CHAGAS $^{4}$, \\ WILSON BARBOSA ${ }^{5}$, IDIANA MARINA DALASTRA ${ }^{6}$, TAILENE ELISA KOTZ ${ }^{2}$
}

RESUMO - O objetivo do presente trabalho foi avaliar a ação do AIB e cianamida hidrogenada no enraizamento de estacas apicais lenhosas de figueira 'Roxo de Valinhos'. Estacas caulinares lenhosas da porção apical dos ramos, coletadas no final da primeira quinzena de julho, foram padronizadas com $20 \mathrm{~cm}$ de comprimento e diâmetro próximo a sete mm. As estacas foram tratada ou não com AIB a $2.000 \mathrm{mg} \mathrm{L}^{-1}$ por 10 seg. e, em seguida, foram enterradas em leito de areia umedecido sob telado constituído de sombrite com $50 \%$ de luminosidade. Imediatamente após o plantio, cinco $\mathrm{cm}$ do ápice das estacas foram pincelados, com diferentes concentrações de cianamida hidrogenada: $0 ; 5 ; 10 ; 15 ; 20$ e $25 \mathrm{~mL} \mathrm{~L}^{-1}$. Passados 60 dias da estaquia, foram mensurados a porcentagem de estacas vivas, enraizadas, brotadas, o número médio das brotações e raízes, além do comprimento médio das brotações. Uma amostragem de cada tratamento foi transplantada para sacolas plásticas com capacidade de $3 \mathrm{~L}(30 \times 18 \mathrm{~cm})$, preenchidas com substrato à base de casca de pínus e foram desbrotadas, preservando apenas uma única brotação por estaca. Aos 30; 60 e 90 dias após o transplantio, mensurou-se o comprimento médio da brotação. Para a melhoria do enraizamento adventícios, emissão e crescimento das brotações em estacas lenhosas apicais de figueira, devem-se aplicar $2.000 \mathrm{mg} \mathrm{L}^{-1}$ de AIB associados com $10 \mathrm{~mL} \mathrm{~L}^{-1}$ de cianamida hidrogenada.

Termos para Indexação: Ficus carica L., ácido indolbutírico e produção de mudas.

\section{ROOTING OF APICAL HARDWOOD CUTTINGS OF FIG TREE 'Roxo de Valinhos' WITH APPLICATION OF IBA AND HYDROGEN CYANAMIDE}

\begin{abstract}
The objective of the present work was to verify the action of IBA and hydrogen cyanamide in the rooting of fig tree apical cuttings 'Roxo de Valinhos'. Woody cutting of apical portion was collected in the end of first fifteen days of July, standardized with $20 \mathrm{~cm}$ of length and seven millimeters of diameter. The cutting was treated or not with $2000 \mathrm{mg} \mathrm{L}^{-1}$ of IBA for 10 seconds. Soon after, the cuttings were buried in bed of sand humidified in nursery conditions with $50 \%$ of brightness. Immediately after the plantation, five $\mathrm{cm}$ of the apex of the cutting was painted with a brush, with different hydrogen cyanamide concentrations: $0,5,10,15,20$ and $25 \mathrm{~mL} \mathrm{~L}^{-1}$. After 60 days, the percentage of alive, rooted, sprouted, the medium number of the sprouting and roots, besides the medium length of the sprouting were evaluated. A sampling of each treatment was transplanted for plastic sacks with capacity of $3 \mathrm{~L}(30 \mathrm{x} 18 \mathrm{~cm})$, filled with substrate the base of pinus peel, preserving a unique sprouting for cutting. The 30, 60 and 90 days after the transplantation, the medium length of the sprouting was evaluated. It was concluded that the cutting apical fig tree 'Roxo de Valinhos' should be treated with $2000 \mathrm{mg} \mathrm{L}^{-1}$ of IBA and $10 \mathrm{~mL} \mathrm{~L}^{-1}$ of hydrogen cyanamide.
\end{abstract}

Index terms: Ficus carica L., indolbutyric acid and seedlings production.

\footnotetext{
(Trabalho 071-08). Recebido em: 25-03-2008. Aceito para publicação em: 04-11-2008.

${ }^{2}$ Acadêmico do curso de Agronomia, Universidade Estadual do Oeste do Paraná - UNIOESTE. Rua Pernambuco, 1.777, Caixa Postal 1.008, Centro, 85960-000, Marechal Cândido Rondon-PR. tatianeohland@hotmail.com taileneelisa@hotmail.com

${ }^{3}$ Eng $^{\circ}$. Agrônomo, D.Sc., Professor Adjunto da Universidade Estadual do Oeste do Paraná - UNIOESTE. Rua Pernambuco, 1.777, Caixa Postal 1.008, Centro, 85960-000, Marechal Cândido Rondon-PR. Bolsista Produtividade em Pesquisa CNPq. Autor para correspondência: rafaelpio@hotmail.com

${ }^{4} E_{n}{ }^{\circ}$. Agrônomo, D.Sc., Pesquisador Científico do Centro Avançado de Pesquisa Tecnológica do Agronegócio de Frutas, Instituto Agronômico - IAC. Av. Luiz Pereira dos Santos, 1.500, Corrupira, 13214-820, Jundiaí-SP. echagas@iac.sp.gov.br ${ }^{5}$ Biólogo, M.Sc., Pesquisador Científico, Centro Experimental Central, Instituto Agronômico - IAC. Caixa Postal 28, 13001-970, Campinas-SP. Bolsista Produtividade em Pesquisa CNPq.wbarbosa@iac.sp.gov.br

${ }^{6} E_{n g}$. Agrônomo, Mestrando do curso de Pós-graduação em Agronomia, Universidade Estadual do Oeste do Paraná UNIOESTE. Rua Pernambuco, 1.777, Caixa Postal 1.008, Centro, 85960-000, Marechal Cândido Rondon-PR. ididalastra@yahoo.com.br
} 
Estacas oriundas da porção apical dos ramos de figueira 'Roxo de Valinhos' vêm demonstrando ser promissoras na propagação da figueira, frente aos bons resultados obtidos com o enraizamento dessa porção do ramo (Pio et al., 2004). Utilização de propágulos de menores dimensões vêm a facilitar o manejo no viveiro, acarretando no aumento da densidade das mudas, frente à utilização de recipientes de menores dimensões. Entretanto, no enraizamento das estacas apicais coletadas ao final do período hibernal (início de agosto), sob telado constituído de sombrite com $50 \%$ de luminosidade, obtiveram-se aproximadamente $49 \%$ de enraizamento e com a aplicação exógena de ácido indolbutírico (AIB), havendo apenas incremento de $8 \%$ (Pio et al., 2006).

Com a introdução de um novo sistema de condução da figueira, por meio de desponte, na obtenção de figos verdes, ocorre a formação de até 96 ponteiros, quando adotados quatro despontes ao longo da condução da figueira em seu ciclo produtivo. Assim, torna-se necessário estudar a possibilidade de enraizamento das estacas apicais, o que decorre em vantagem ao maior aproveitamento dos propágulos a serem multiplicados (Gonçalves et al., 2006; Alvarenga et al., 2007).

O grupo de fitorreguladores utilizado com maior frequiência no enraizamento adventício em estacas é o das auxinas (Hinojosa, 2000). Uma das formas mais estudadas no favorecimento do balanço hormonal para o enraizamento é a aplicação exógena do fitorregulador ácido indolbutírico (AIB), por tratarse de uma substância fotoestável, de ação localizada e menos sensível à degradação biológica, em comparação às demais auxinas sintéticas, podendo, em muitas espécies de difícil enraizamento, viabilizar a produção de mudas por meio da estaquia (Fachinello et al., 2005).

A cianamida hidrogenada $\left(\mathrm{H}_{2} \mathrm{CN}_{2}\right)$ pode ser utilizada para o suprimento artificial da endodormência das gemas (Shulman et al., 1986; George \& Nissen, 1993). Sabe-se que a cianamida hidrogenada é rapidamente absorvida e metabolizada, sua ação causa diminuição da atividade da catalase, sem modificar a peroxidase, o que resulta em um aumento da concentração de peróxido $\left(\mathrm{H}_{2} \mathrm{O}_{2}\right)$ nas gemas (Shulman et al., 1986; Goldback et al., 1988). Este aumento é responsável pela ativação do ciclo das pentoses e conseqüente indução da saída da dormência das gemas (Omran, 1980).

Estudos mostram a necessidade de determinado equilíbrio entre as auxinas e os hidratos de carbono para o sucesso do enraizamento (Bhattacharya et al., 1985; Wang \& Andersen, 1989).
Mindêllo Neto (2006), trabalhando apenas com avaliações pertinentes à rizogênese de estacas de ameixeiras, verificou que a aplicação de $0,5 \mathrm{~mL} \mathrm{~L}^{-1} \mathrm{de}$ cianamida hidrogenada aumentou a porcentagem de enraizamento de estacas lenhosas de ameixeira 'Amarelinha' em 4,2\%.

Pressupõe-se que a submissão da base de estacas em AIB, associada à aplicação de cianamida hidrogenada no ápice das estacas, favoreça o enraizamento e a brotação das gemas, vindo a contribuir em incrementos significativos, tanto na rizogênese, como no intumescimento precoce das gemas, e sucessiva brotação. Como as auxinas são sintetizadas principalmente em gemas apicais e, de maneira geral, movem-se através do ápice para a base (Hartmann et al., 2002), o estímulo da ação da cianamida hidrogenada na quebra da dormência das gemas localizadas no ápice das estacas apicais de figueira, poderá apresentar sinergismo à aplicação exógena do AIB, aumentando consideravelmente a rizogênese na estaca.

Assim, o objetivo do presente trabalho foi mencionar o potencial rizogênico e de brotação de estacas apicais lenhosas de figueira 'Roxo de Valinhos', submetidas à aplicação exógena de AIB e cianamida hidrogenada.

Estacas apicais lenhosas de ramos da figueira 'Roxo de Valinhos' foram coletadas no final do período hibernal (julho), em um pomar comercial situado no município de Quatro Pontes, região oeste do Estado do Paraná. Em seguida, foram transportadas ao Setor de Fruticultura, da Universidade Estadual do Oeste do Paraná (UNIOESTE), Marechal Cândido Rondon-PR, para a execução do experimento.

As estacas foram padronizadas com $20 \mathrm{~cm}$ de comprimento, diâmetro próximo a sete $\mathrm{mm}$, sendo efetuado um corte em bisel na base da estaca e outro reto no ápice. Metade da base das estacas foi tratada em solução de AIB a $2.000 \mathrm{mg} \mathrm{L}^{-1}$, por $10 \mathrm{~s}$, e a outra metade permaneceu sem tratamento. Posteriormente, as estacas foram enterradas a $2 / 3$ de seu comprimento em leito de areia umedecido, sob telado constituído de sombrite com $50 \%$ de luminosidade. Imediatamente após o plantio, $5 \mathrm{~cm}$ do ápice das estacas foram tratados, com auxílio de um pincel de cerdas finas, com diferentes concentrações de cianamida hidrogenada: $5 ; 10 ; 15 ; 20$ e $25 \mathrm{~mL} \mathrm{~L}^{-1}$, além da testemunha, pincelada apenas com água. Durante a fase experimental, o leito de enraizamento foi racionalmente umedecido.

O delineamento utilizado foi o inteiramente casualizado, no esquema fatorial $2 \times 6$, sendo o primeiro fator constituído por tratamento ou não com 
AIB, e o segundo fator, pelas concentrações de cianamida hidrogenada, com quatro repetições e 10 estacas por unidade experimental. Passados 60 dias da estaquia, foi mensurada a porcentagem de estacas vivas, enraizadas, brotadas, o número médio das brotações e raízes, além do comprimento médio das brotações.

Uma amostra de cinco estacas por parcela foi transplantada para sacolas plásticas com capacidade de $3 \mathrm{~L}(30 \times 18 \mathrm{~cm})$, preenchidas com substrato à base de casca de pínus, sendo as estacas desbrotadas, preservando apenas uma única brotação por estaca e permaneceram dentro do mesmo telado. Aos 30; 60 e 90 dias após o transplantio, mensurou-se o comprimento médio da brotação.

Os dados foram submetidos à análise de variância, as médias comparadas pelo teste Tukey, ao nível de $5 \%$ de probabilidade, e as concentrações de cianamida hidrogenada, submetidas à regressão, segundo as recomendações de Gomes (2000). As análises foram realizadas pelo programa computacional Sistema para Análise de Variância SISVAR (Ferreira, 2000).

Segundo a análise estatística dos dados, não houve interação entre as concentrações de AIB e cianamida hidrogenada em nenhuma das variáveis mensuradas.

A submissão da base das estacas apicais de figueira, na concentração de $2.000 \mathrm{mg} \mathrm{L}^{-1}$ de AIB, aumentou a porcentagem de sobrevivência das estacas $(6,6 \%)$, de enraizamento $(27,4 \%)$ e significativamente o número médio de raízes, com o expressivo aumento de 84,2 raízes, em comparação à testemunha, sem tratamento com AIB (Tabela 1). O aumento do número de raízes por estacas contribui na operação de transplantio das estacas para sacolas plásticas, minimizando as perdas em possíveis quebras de raízes na operação de transplantio e ainda refletindo positivamente no desenvolvimento da brotação e do sistema radicular. Esses resultados concordam com os relatados por Pio et al. (2006), os quais conseguiram acréscimo de rizogênese das estacas apicais de figueira com a utilização de $2.000 \mathrm{mg} \mathrm{L}^{-1} \mathrm{AIB}$, em comparação com a ausência do tratamento.

Além da melhoria na rizogênese, nas estacas apicais, a aplicação da concentração de AIB testada ainda auxiliou no aumento do número e no comprimento médio de brotações. Essa ação secundária do AIB, em relação aos brotos das estacas, pode estar relacionada à melhoria expressiva na rizogênese, principalmente quanto ao aumento do número de raízes, o que ocasionou aumento na taxa respiratória da estaca e desencadeou a mobilização das reservas (carboidratos), favorecendo a emissão das brotações.

Quanto à aplicação da cianamida hidrogenada no ápice das estacas, não houve diferença estatística para a porcentagem de estacas vivas e número médio de brotações. Aplicações crescentes de cianamida hidrogenada promoveram decréscimo linear na porcentagem de estacas enraizadas, obtendo-se $73,22 \%$ de enraizamento na ausência de tratamento, queda desse percentual em 19,9\% com a utilização de $25 \mathrm{~mL} \mathrm{~L}^{-1}$ (Figura 1). Porém, as perdas ocorridas com as concentrações de $5 \mathrm{~mL} \mathrm{~L}^{-1}(3,98 \%)$ e $10 \mathrm{~mL} \mathrm{~L}^{-1}$ $(7,96 \%)$ de cianamida hidrogenada foram mínimas em comparação com a testemunha. A queda na porcentagem de enraizamento em função das aplicações crescentes de cianamida hidrogenada está associada à ação dessa substância nas brotações das estacas, uma vez que foi observado, na primeira quinzena experimental, o aumento expressivo das brotações nas estacas tratadas com cianamida hidrogenada, em comparação com as não-tratadas, ocorrendo antecipação na emissão das brotações. Esse fato certamente preconizou a mobilização das reservas, inicialmente na emissão dos brotos, desfavorecendo, em primeira estância, a rizogênese.

A cianamida hidrogenada é utilizada na superação da dormência das gemas sendo rapidamente absorvida e metabolizada, ocasionando a diminuição da atividade da catalase, aumentando a concentração de água oxigenada e consequente indução da quebra de dormência das gemas (Omran, 1980; Shulman et al., 1986; Goldback et al., 1988).

Isso foi comprovado na mensuração da porcentagem de estacas brotadas e no comprimento médio das brotações. Pela curva da regressão da porcentagem de estacas brotadas, verifica-se que houve aproximadamente $80 \%$ de brotação, nas estacas tratadas com $10 \mathrm{~mL} \mathrm{~L}^{-1}$ e $15 \mathrm{~mL} \mathrm{~L}^{-1}$ de cianamida hidrogenada, aumento de $11,27 \%$ em comparação com a ausência do tratamento (Figura 2). A mesma tendência ocorreu na curva da regressão do comprimento médio das brotações, ou seja, as brotações apresentaram, em média, aproximadamente $29 \mathrm{~mm}$, nas estacas tratadas com $10 \mathrm{mLL}^{-1} \mathrm{e} 15 \mathrm{mLL}^{-1} \mathrm{de}$ cianamida hidrogenada, aumento de 50\% em relação à testemunha (14,1 mm) (Figura 3).

Em consequência da ação da cianamida hidrogenada na emissão das brotações, houve efeito secundário na emissão de raízes na base das estacas, o que pode ser constatado na Figura 4, obtendo-se aproximadamente 55 raízes, em média, com a utilização de $15 \mathrm{~mL} \mathrm{~L}^{-1}$ de cianamida hidrogenada, 20 raízes a mais que a ausência de tratamento. 
Como a aplicação da cianamida hidrogenada foi na porção apical das estacas e sua ação está diretamente ligada às gemas, o gasto de reserva (carboidratos) na emissão e elongação das brotações desfavoreceu a porcentagem de enraizamento das estacas que receberam o tratamento; no entanto; com o aumento do metabolismo das gemas, pode ter favorecido a produção de ácido indolacético (AIA), substância endógena responsável pela rizogênese e, consequentemente, aumento na emissão de raízes.

O enraizamento de estacas é influenciado por substâncias hormonais localizadas endogenamente nas estacas. As auxinas são as responsáveis pelo enraizamento, dentre as quais destaca-se o AIA, produzido nas regiões de crescimento, principalmente as gemas (Hinojosa, 2000). As auxinas estimulam a divisão celular, além de apresentarem relações bastante importantes com ácidos nucleicos e proteínas, modificações da parede celular e estimulação de atividades enzimáticas (Hartmann et al., 2002). Entre as principais funções biológicas das auxinas, pode-se citar o crescimento de órgãos, especialmente as raízes (Haissig, 1996).

Outra razão é que a aplicação de cianamida hidrogenada, de certa forma, contribui para aumentar os níveis de carboidratos e nitrogênio, auxiliando na emissão de raízes (Wang \& Andersen, 1989; Ono \& Rodrigues, 1996). Aimportância dos carboidratos para formação de raízes é que a auxina requer fonte de carbono para a biossíntese de ácidos nucleicos e proteínas, levando à necessidade de energia e carbono para a formação das raízes (Hartmann et al., 2002).

TABELA 1- Porcentagem de estacas vivas (PEV), enraizadas (PEE), brotadas (PEB), número médio de brotações (NMB) e raízes (NMR) e comprimento médio das brotações (CMB, mm) de estacas apicais de figueira 'Roxo de Valinhos', submetidas ou não ao tratamento com $\operatorname{AIB}\left(2.000 \mathrm{mg} \mathrm{L}^{-1}\right)$. Marechal Cândido Rondon-PR, UNIOESTE, 2008.

\begin{tabular}{llllllll}
\hline Tratamento & \multicolumn{7}{c}{ Variáveis analisadas* } \\
\cline { 2 - 7 } & PEV & PEE & PEB & NMB & NMR & CMB (mm) \\
\hline Sem AIB & $90,4 \mathrm{~b}$ & $50,0 \mathrm{~b}$ & 75,8 ns & $1,4 \mathrm{~b}$ & 10,4 & $\mathrm{~b}$ & 12,0 \\
Com AIB $\left(2.000 \mathrm{mg} \mathrm{L}^{-1}\right)$ & $97,0 \mathrm{a}$ & $77,4 \mathrm{a}$ & 81,7 & $2,1 \mathrm{a}$ & $94,6 \mathrm{a}$ & $27,1 \mathrm{a}$ \\
\hline C.V.(\%) & 7,7 & 23,1 & 17,6 & 16,1 & 21,9 & 13,4 \\
\hline
\end{tabular}

* Médias seguidas pela mesma letra na coluna não diferem significativamente entre si, pelo teste Tukey, ao nível de 5\% de probabilidade. ${ }^{\text {ns }}$ - não-significativo, segundo o teste F.

TABELA 2- Comprimento médio das brotações ( $\mathrm{mm}$ ) de estacas apicais de figueira 'Roxo de Valinhos', submetidas ou não ao tratamento com AIB (2.000 $\left.\mathrm{mg} \mathrm{L}^{-1}\right)$, aos 30; 60 e 90 dias após o transplantio. Marechal Cândido Rondon-PR, UNIOESTE, 2008.

\begin{tabular}{llcc}
\hline Tratamento & \multicolumn{3}{c}{ Comprimento médio das brotações $(\mathrm{mm}){ }^{*}$} \\
\cline { 2 - 4 } & 30 dias & 60 dias & 90 dias \\
\hline Sem AIB & $33,5^{\mathrm{ns}}$ & $100,5^{\mathrm{ns}}$ & $128,0^{\mathrm{ns}}$ \\
Com AIB $\left(2000 \mathrm{mg} \mathrm{L}^{-1}\right)$ & 38,5 & 96,3 & 118,0 \\
\hline C.V.(\%) & 28,72 & 15,31 & 14,20 \\
\hline
\end{tabular}

* Médias seguidas pela mesma letra na coluna não diferem significativamente entre si, pelo teste Tukey, ao nível de 5\% de probabilidade. $^{\text {ns }}$ - não-significativo, segundo o teste F. 


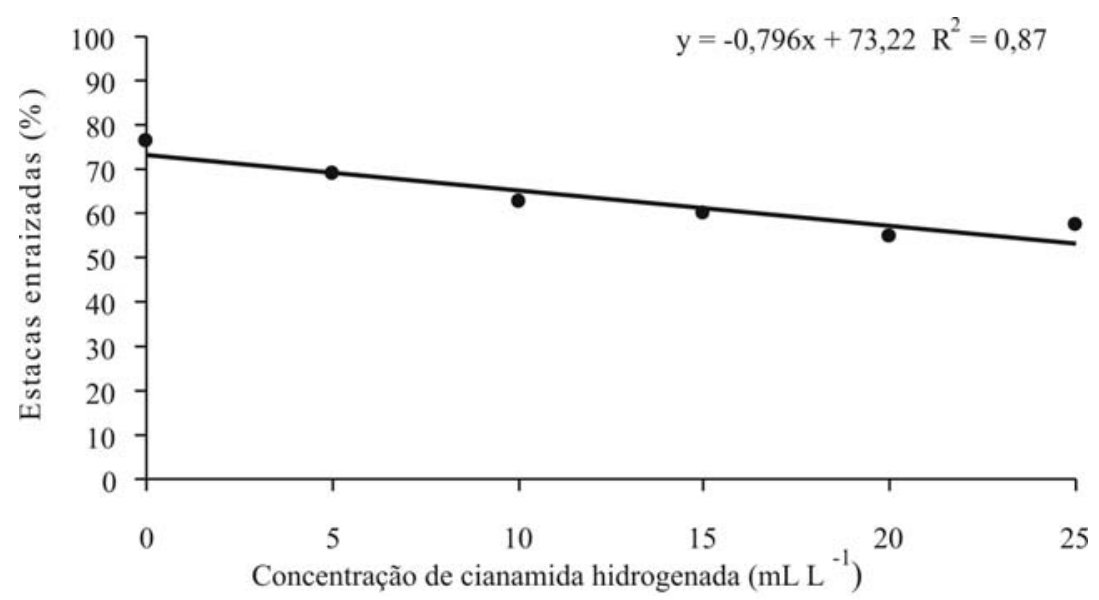

FIGURA 1- Porcentagem de estacas apicais enraizadas de figueira 'Roxo de Valinhos' submetidas a diferentes concentrações de cianamida hidrogenada. Marechal Cândido Rondon-PR, UNIOESTE, 2008.

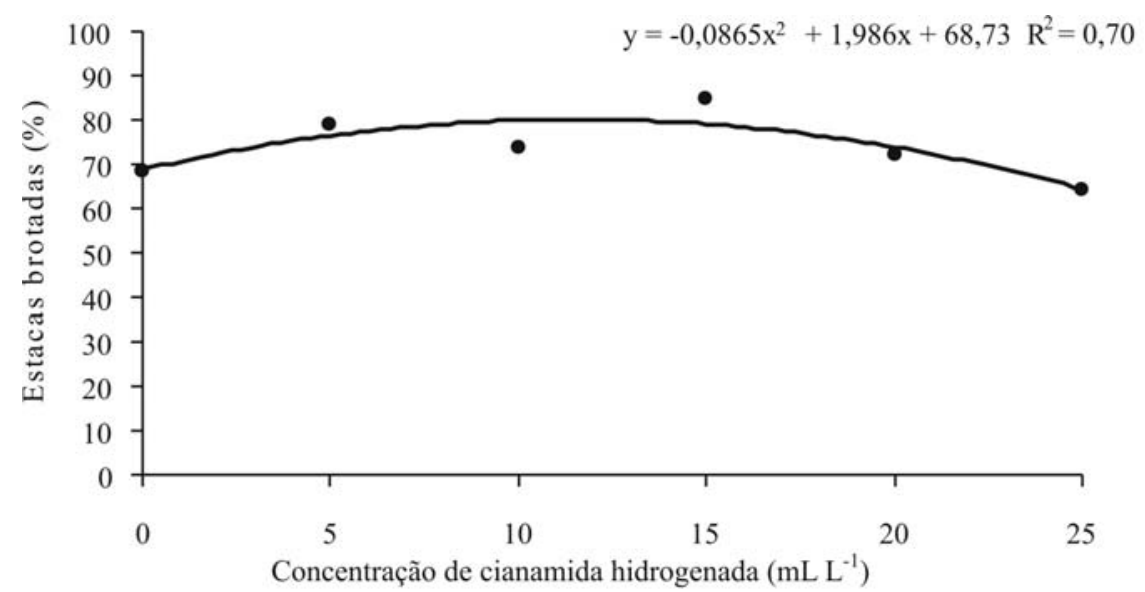

FIGURA 2- Porcentagem de estacas apicais brotadas de figueira 'Roxo de Valinhos' submetidas a diferentes concentrações de cianamida hidrogenada. Marechal Cândido Rondon-PR, UNIOESTE, 2008.

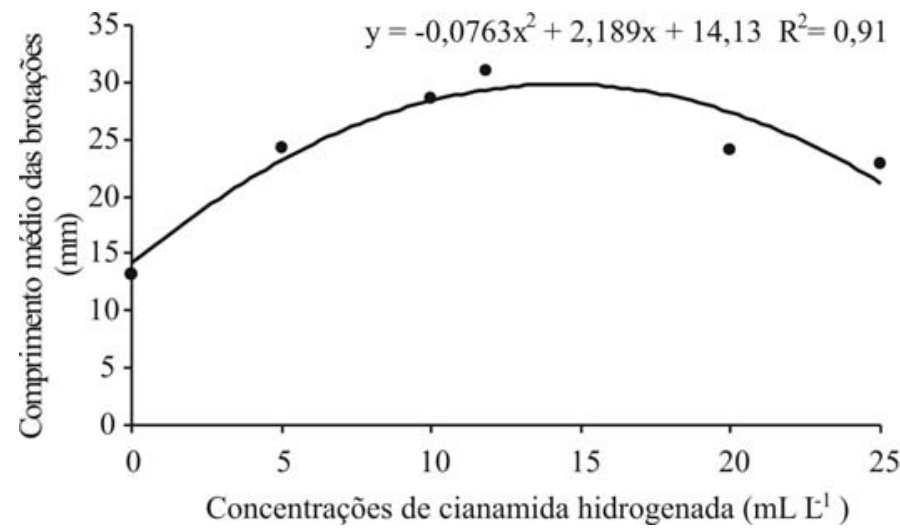

FIGURA 3- Comprimento médio das brotações de estacas apicais enraizadas de figueira 'Roxo de Valinhos' submetidas a diferentes concentrações de cianamida hidrogenada. Marechal Cândido RondonPR, UNIOESTE, 2008. 


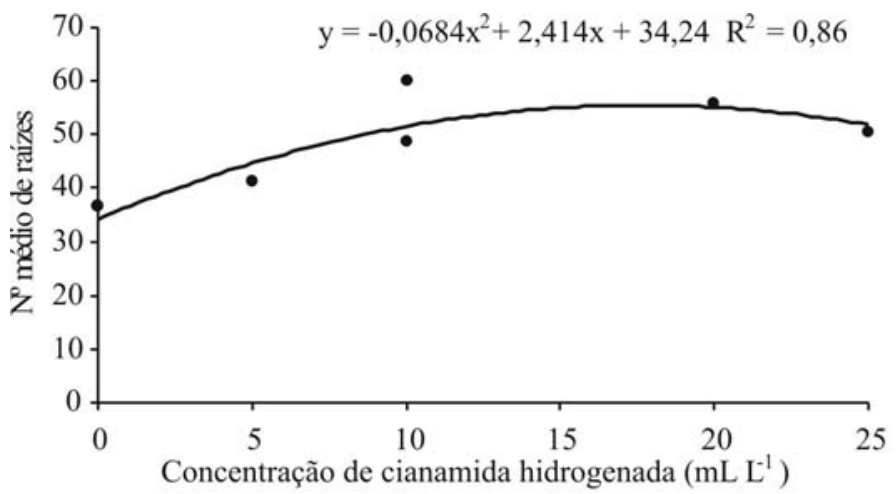

FIGURA 4- Número médio de raízes de estacas apicais enraizadas de figueira 'Roxo de Valinhos' submetidas a diferentes concentrações de cianamida hidrogenada. Marechal Cândido Rondon-PR, UNIOESTE, 2008.

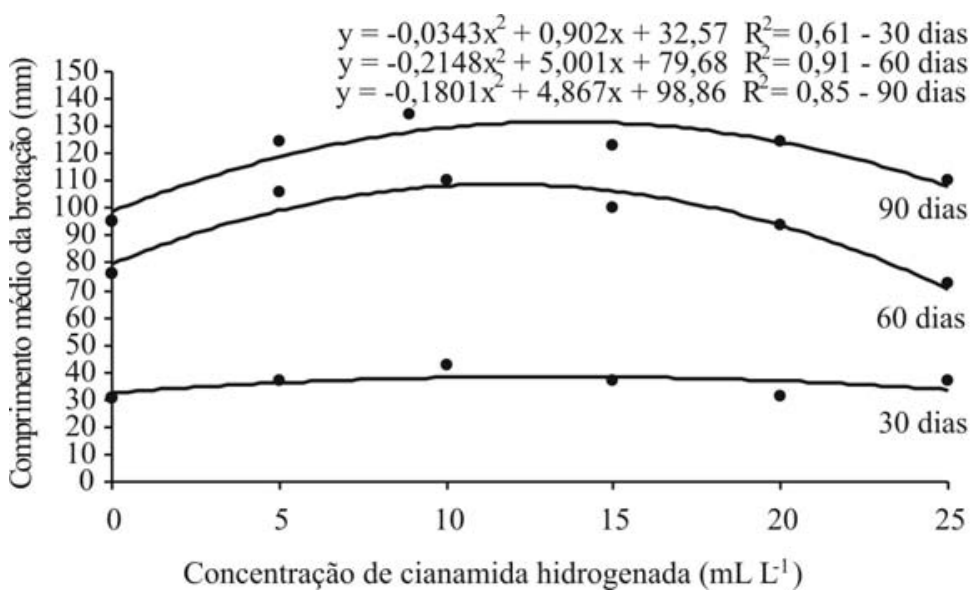

FIGURA 5- Comprimento médio das brotações de estacas apicais enraizadas de figueira 'Roxo de Valinhos' submetidas a diferentes concentrações de cianamida hidrogenada, aos 30; 60 e 90 dias após o transplantio. Marechal Cândido Rondon- PR, UNIOESTE, 2008.

\section{REFERÊNCIAS}

ALVARENGA, A.A.; ABRAHAO, E.; FRAGUAS, J.C.; CARVALHO, V.L.; SILVA, R.A.; SANTA CECILIA, L.V.C.; CUNHA, R.L.; SILVA, V.J. Figo (Ficus carica L.). In: TRAZILBO, J.P.Jr.; MADELAINE V. (Org.). 101 Culturas: manual de tecnologias agrícolas. Belo Horizonte: EPAMIG, 2007. p.365-372.

BHATTACHARYA, S.; BHATTACHARYA, N.C.; STRAIN, B.R. Rooting of sweet potato stem cuttings under $\mathrm{CO} 2$ enriched environment and with IAA treatment. Hortscience, Alexandria, v.20, p.1109-1110, 1985.

FACHINELLO, J.C.; HOFFMANN, A.; NACHTIGAL, J.C. Propagação de plantas frutíferas. Brasília: Embrapa, 2005. 221p.
FERREIRA, D.F. Análise estatística por meio do SISVAR (Sistema para Análise de Variância) para Windows versão 4.0. In: REUNIÃO ANUAL DA REGIÃO BRASILEIRA DA SOCIEDADE INTERNACIONAL DE BIOMETRIA, 45., 2000, São Carlos. Anais... São Carlos: UFSCar, 2000. p.255-258.

GEORGE, A.P.; NISSEN, R.J. Effects of growth regulants on defoliation, flowering, and fruit maturity of the low chill peach cultivar Flordaprince in subtropical Australia. Australian Journal of Experimental Agriculture, Collingwood, v.33, p.787795, 1993.

GOLDBACK, H.; THALER, C.; WÜNSCH, A. Decomposition of 14C- labeled cyanamide in Vitis vinifera cuttings. Journal of Plant Physiology, Elsevier, v.133, p.299-303, 1988. 
GOMES, F.P. Curso de estatística experimental. 14 ed. Piracicaba: USP/ESALQ, 2000. 477p.

GONÇALVES, C.A.A.; LIMA, L.C.O.; LOPES, P.S.N.; SOUZA, M.T. Poda e sistemas de condução na produção de figos verdes. Pesquisa Agropecuária Brasileira, Brasília, v.41, n.6, p.955-961, 2006.

HAISSIG, B.E. Metabolic process in adventitious rooting of cuttings. In: JACKSON, M. B. (Ed.). New root formation in plants and cuttings. Dordrecht: Matinus Nijhoff, 1996. p.141-190.

HARTMANN, H.T.; KESTER, D.E.; DAVIES JUNIOR, F.T.; GENEVE, R.L. Plant propagation: principles and practices. $7^{\text {th }}$ ed. New Jersey: Prentice Hall, 2002. 880p.

HINOJOSA, G.F. Auxinas. In: CID, L.P.B. Introdução aos hormônios vegetais. Brasília: EMBRAPA, 2000. p.15-54.

MINDÊLLO NETO, U.R. Enraizamento de estacas lenhosas de ameixeira com aplicação de ácido indolbutírico e cianamida hidrogenada. Pesquisa Agropecuária Brasileira, Brasília, v.41, n.3, p.529$531,2006$.

OMRAN, R.G. Peroxide levels and the activities of catalase, peroxidase, and indoleacetic acid oxidase during and after chilling of cucumber seedings. Plant Physiology, Rockville, v.65, p.407-408, 1980.
ONO, E.O.; RODRIGUES, J.D. Aspectos da fisiologia do enraizamento de estacas caulinares. Botucatu: Unesp/Funep, 1996. 83p.

PIO, R.; RAMOS, J.D.; CHALFUN, N.N.J.; COELHO, J.H.C.; GONTIJO, T.C.A.; CARRIJO, E.P.; VILLA, F. Enraizamento adventícios de estacas apicais de figueira e desenvolvimento inicial das plantas no campo. Ciência e Agrotecnologia, Lavras, v.28, n.1, p.215-221, 2004.

PIO, R.; RAMOS, J.D.; CHALFUN, N.N.J.; GONTIJO, T.C.A.; MENDONÇA, V.; CARRIJO, E.P.; CHAGAS, E.A. Propagação de estacas apicais de figueira: diferentes ambientes, ácido indolbutírico e tipo de estaca. Ciência e Agrotecnologia, Lavras, v.30, n.5, p.1021-1026, 2006.

SHULMAN, Y.; NIR, G.; LAVEE, S. Oxidative processes in bud dormancy and the use of hydrogen cyanamide in breaking dormancy. Acta Horticulturae, Wageningen, v.179, p.141-148, 1986.

WANG, Q.; ANDERSEN, A.S. Propagation of Hibiscus rosasinensis: relations between stock plant cultivar age, environment and growth regulator treatments. Acta Horticulturae, Wageningen, v.251, p.289-309, 1989. 